\title{
Field dispersal of the parasitoid wasp Habrobracon hebetor (Hymenoptera: Braconidae) following augmentative release against the millet head miner Heliocheilus albipunctella (Lepidoptera: Noctuidae) in the Sahel
}

Ibrahim Boukary Baoua, Malick N. Ba, Laouali Amadou, Adama Kabore \& Clementine L. Dabire-Binso

To cite this article: Ibrahim Boukary Baoua, Malick N. Ba, Laouali Amadou, Adama Kabore \& Clementine L. Dabire-Binso (2018): Field dispersal of the parasitoid wasp Habrobracon hebetor (Hymenoptera: Braconidae) following augmentative release against the millet head miner Heliocheilus albipunctella (Lepidoptera: Noctuidae) in the Sahel, Biocontrol Science and Technology, DOI: 10.1080/09583157.2018.1450842

To link to this article: https://doi.org/10.1080/09583157.2018.1450842

册 Published online: 15 Mar 2018.

Submit your article to this journal $₫$

凹 Article views: 8

Q View related articles ๘

View Crossmark data $\nearrow$ 


\title{
Field dispersal of the parasitoid wasp Habrobracon hebetor (Hymenoptera: Braconidae) following augmentative release against the millet head miner Heliocheilus albipunctella (Lepidoptera: Noctuidae) in the Sahel
}

\author{
Ibrahim Boukary Baoua ${ }^{a}$, Malick N. Ba ${ }^{b}$, Laouali Amadou', Adama Kabore ${ }^{\mathrm{d}}$ and \\ Clementine L. Dabire-Binso ${ }^{d}$
}

\begin{abstract}
${ }^{a}$ Faculty of Agronomy and Environmental Science, Université DanDicko Dankoulodo de Maradi, Maradi, Niger; ${ }^{\mathrm{b}}$ International Institute of Tropical Agriculture, Dryland Cereals, Niamey, Niger; Institut National de Recherches Agronomiques du Niger (INRAN), Maradi, Niger; 'Institut de I'Environnement et de Recherches Agricoles (INERA), Kamboinse, Burkina Faso
\end{abstract}

\begin{abstract}
Pearl millet is one of the major staple food crops in Sub-Sahelian Africa, and the millet head miner (MHM) [Heliocheilus albipunctella] is its major pest, causing serious economic damage in the maturity period. We studied the dispersion patterns of the endogenous ectoparasitoid, Habrobracon hebetor (Hymenoptera: Braconidae), after augmentative releases in pearl millet fields for biological control of the MHM, in 2010 and 2011 in Burkina Faso and Niger. The parasitoids were released using 15 jute bags per release site. Parasitoid dispersion was indirectly monitored through weekly assessments of MHM parasitism by $\mathrm{H}$. hebetor at different distances from release points $(0,3$ and $5 \mathrm{~km})$ and in control villages $(15 \mathrm{~km})$. Our findings indicate that the jute bags released approximately 900-1000 parasitoids per site over a period of three weeks. This initial parasitoid population led to higher parasitism of MHM larvae at the site of dissemination compared to farms at distances of 3 and $5 \mathrm{~km}$. However, usually after five weeks, successive generations of $H$. hebetor dispersed up to $3 \mathrm{~km}$, causing high levels of MHM larval mortality, which sometimes is similar to those of the release points. Based on these results, we recommend the release of parasitoids at sites spaced $3 \mathrm{~km}$ for timely and more efficient control of MHM populations.
\end{abstract}

\section{ARTICLE HISTORY}

Received 11 August 2017

Returned 6 March 2018

Accepted 6 March 2018

\section{KEYWORDS}

Biological control; parasitoid bag; on-farm releases;

Corcyra cephalonica; Burkina Faso; Niger

\section{Introduction}

The millet head miner (MHM), Heliocheilus albipunctella de Joannis (Lepidoptera: Noctuidae), is one of the most devastating insect pests of pearl millet (Pennisetum glaucum) in the Sahel (Gahukar, 1984). This important univoltine pest has a geographical distribution between latitudes of $11^{\circ} \mathrm{N}$ and $15^{\circ} \mathrm{N}$ within the Southern Sahel and Sudan bioclimatic zones (Nwanze \& Sivakumar, 1990). The MHM can cause major yield 
losses, especially of early maturing pearl millet (Krall, Youm, \& Kogo, 1995; Nwanze \& Sivakumar, 1990; Youm \& Owusu, 1998). Common pest control methods such as cultural control practices, host plant resistance, and spraying insecticides are ineffective or impractical (Gahukar, 1989, 1990, 1992; Nwanze \& Sivakumar, 1990). Biological control has emerged as the most attractive solution for controlling the MHM, and the ectolarval parasitoid Habrobracon hebetor Say is considered a natural enemy with a great potential (Gahukar et al., 1986). The effectiveness of this parasitoid against the MHM was first demonstrated in the late 1980s in Senegal (Bhatnagar, 1987, 1989), and subsequently in Niger in the early 2000s (Garba \& Gaoh, 2008). In recent years, its effectiveness for augmentative biological control has been demonstrated in the Sahel (Ba et al., 2014, 2013; Kabore, Ba, Dabire-Binso, \& Sanon, 2017; Payne et al., 2011), with an estimated yield gain of 34\% (Baoua et al., 2014). The parasitoids are released from $15 \mathrm{~cm} \times 25 \mathrm{~cm}$ small jute bags containing a mixture of millet grains and flour together with 25 larvae of the rice meal moth, Corcyra cephalonica (Stainton) (Lepidoptera: Pyralidae), and two mated $H$. hebetor females (Ba et al., 2014, 2013). Then, two females parasitise the 25 C. cephalonica host larvae, and emerging progeny escape through the jute mesh and disperse to parasitize MHM larvae in millet fields (Ba et al., 2014, 2013). Thus, the current release protocol suggests the use of 15 parasitoid bags for coverage of a $5 \mathrm{~km}^{2}$ area of pearl millet. This estimate was based on the early recommendations of Garba and Gaoh (2008) but lacked a scientific basis. The release of parasitoids at every $5 \mathrm{~km}$ was implemented in our recent work in the region, but evidence of the efficiency of the augmentative biological programme against the MHM was always based on observations made in farms located in villages where parasitoids were released (Ba et al., 2014, 2013).

To succeed in augmentative biological control programmes, it is crucial to understand the number of natural enemies that should be released per area, and knowledge of the mobility and dispersal capacity of $H$. hebetor is essential for the development of appropriate release protocols. The high levels of parasitism resulting from previous studies suggest that the augmentative releases were successful at increasing the degree of mortality by indigenous $H$. hebetor. Understanding how these parasitoids increase and spread can help in understanding factors important to successful biological control through augmentation. In current augmentative programmes, the objective is to quickly reach the MHM larvae before significant crop damages occur; to accomplish this, the $H$. hebetor parasitoids must disperse from the release points and spread throughout the infested area. To rationalise the current biological control programmes, it is crucial to not only have empirical estimates of the number of $H$. hebetor to be released but also to know how many sites per release area should be considered. The aim of this work was to evaluate the dispersal ability of released $H$. hebetor adults and parasitism rates of MHM after augmentative release and to determine the release protocol that provides the highest parasitoid efficiency.

\section{Materials and methods}

\section{Study location}

The experimental trials were conducted in typical pearl millet production areas of the Sahel agroecological zone of Burkina Faso and Niger in 2010 and 2011. This agroecosystem has a unimodal rainfall pattern with the rainy season extending from June to October, 
and annual rainfall varying between 450 and $550 \mathrm{~mm}$. The maximum relative humidity reaches $92 \%$ during the millet season, and temperatures range from $24^{\circ} \mathrm{C}$ to $39^{\circ} \mathrm{C}$ during the rainy season. During this period, the area has continuous pearl millet fields covering almost $80 \%$ of the cultivated area usually in association with cowpea, while uncultivated land is left for grazing livestock. Farmers grow 2-3 local pearl millet varieties planted in May-June at densities ranging from 4000 to 6000 plants/hills per ha on 3-10 ha. No irrigation or chemical fertiliser is applied to any of the fields.

\section{Parasitoid rearing}

A $H$. hebetor colony was established from field-collected MHM larvae and maintained in the laboratory at room temperature $\left(\right.$ mean $=26 \pm 2^{\circ} \mathrm{C}$ ) on the rice meal moth, C. cephalonica, at the National Agricultural Research Institutes of Burkina Faso and Niger. Fieldcaught insects were added to the colonies once per year. Rice moths were reared on a mixture of pearl millet grain and flour in wooden cages $(20 \times 20 \times 13 \mathrm{~cm})$, and the parasitoids were reared on third and fourth instar C. cephalonica larvae using a technique described by Ba et al. (2014, 2013).

\section{Assessing initial numbers of parasitoids emerging from bags placed in millet fields}

This experiment was carried out in 2010 and included 6 villages in each country, each supplied with 10 parasitoid jute bags $(25 \mathrm{~cm} \times 15 \mathrm{~cm})$. Each parasitoid bag contained $500 \mathrm{~g}$ of millet, 25 C. cephalonica larvae and two mated $H$. hebetor females. The parasitoid-containing bags were placed within millet farms ( 2 bags/farm) and covered with muslin cloth to trap the emerging parasitoids. Bags were kept in the field for 1 month, and emerging parasitoids were sexed, counted and removed daily.

Based on the initial number of parasitoid-inoculated hosts from each bag, we estimated the population of $H$. hebetor build-up over the four weeks in the field using the published $H$. hebetor life tables on MHM larvae (Youm \& Gilstrap, 1993a). The same data were used to estimate the total MHM larvae population killed/parasitised.

\section{Assessing field dispersal of parasitoids after on-farm releases}

This experiment was carried out in 2010 with a group of four villages in Burkina Faso and four villages in Niger selected for endemic infestations with the MHM with over $60 \%$ of panicles infested. In each country, the four villages were located at least $25 \mathrm{~km}$ apart. Each of the four villages was supplied with 15 parasitoid bags as described above, evenly distributed among five millet farms (three bags/farm) using methods described by $\mathrm{Ba}$ et al. (2014). Dispersal of parasitoids was monitored from release points following transects in wind and crosswind directions. Observation points were located at farms $0 \mathrm{~km}$ and $3 \mathrm{~km}$ from parasitoid released points and in control, no-release villages located $15 \mathrm{~km}$ away from release points.

At each observation point, 200 pearl millet panicles were randomly collected. The data were collected weekly, starting on the day of parasitoid bag establishment and ending five weeks later. Parasitoid dispersal was indirectly assessed by counting the numbers of live 
(unparasitised) and $H$. hebetor-parasitised MHM larvae. Larvae parasitised by $H$. hebetor were easily distinguished by the presence of cocoons (Garba \& Gaoh, 2008).

The same experiment was repeated in 2011 in another set of four villages in Burkina Faso and Niger. The villages were selected using some of the characteristics listed above. In this experiment, the parasitoids were released in each of the four villages, and dispersal of parasitoids was monitored at 0,3 and $5 \mathrm{~km}$ from release villages and in control villages located $15 \mathrm{~km}$ away from release points using the methods described above.

\section{Data analysis}

Data for MHM parasitism by $H$. hebetor in wind and crosswind directions was pulled for each location and compared using Analysis of Variance (ANOVA PROC GLM) with SAS software version 9.1 (SAS, 2003). When ANOVAs were significant, means were compared by the Student-Newman-Keuls tests at the 5\% level. Percentages from parasitism data were arcsine transformed prior to statistical tests.

\section{Results}

\section{Parasitoid emergence from jute bags}

When parasitoid bags were deployed on the millet farms, the parasitoid progeny began emerging from the bags 8 days after confinement of $H$. hebetor females with $C$. cephalonica larvae. The emergence period lasted 7 days in Burkina Faso, while it extended to 12 days in Niger (Figure 1). Up to 70 parasitoids emerged from the bags in Burkina Faso, while only 60 emerged from the bags in Niger. The emerging parasitoids had a sex ratio of 0.72 (female/male) in Burkina Faso and 0.57 (female/male) in Niger. The estimated numbers

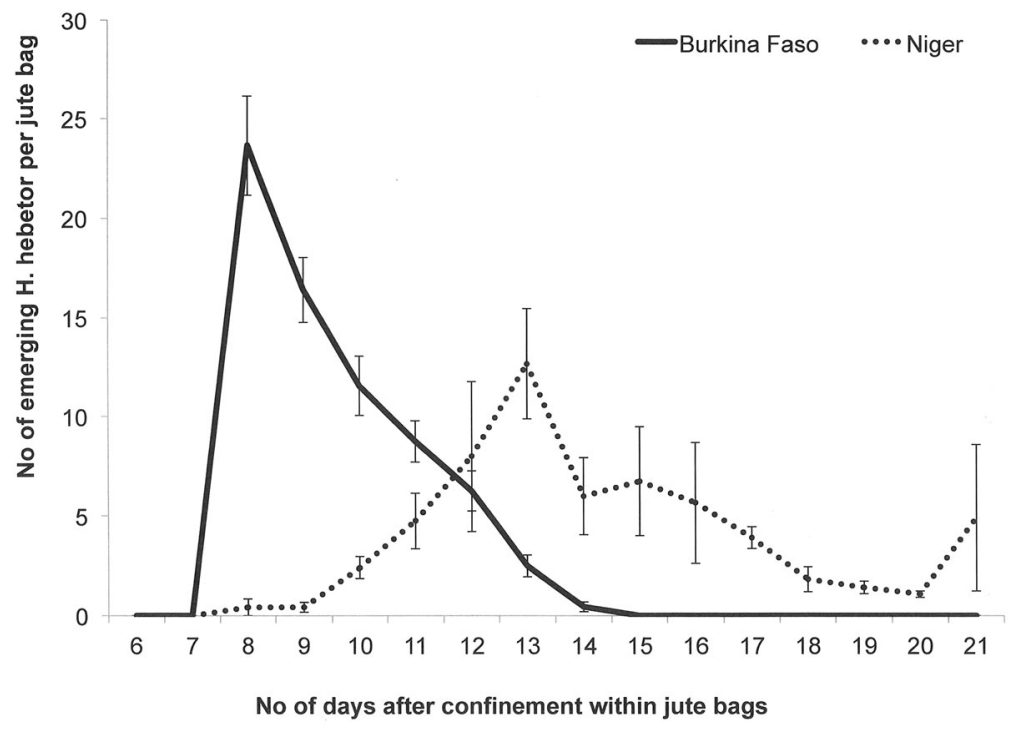

Figure 1. Daily emergence of Habrobracon hebetor (Say) (mean \pm SE) from jute bags deployed in pearl millet fields in Burkina Faso and Niger. 
of $H$. hebetor female population build-up from the inoculum ranged between 960,000 and 1.4 million after one and half months (Figure 2). This led to a cumulative parasitisation of potentially 465,000-697,000 MHM larvae (Figure 2).

\section{Parasitism of the MHM with $\mathrm{H}$. hebetor}

In Burkina Faso in 2010, parasitism of the MHM by H. hebetor was first observed at experimental sites two weeks after parasitoid bags were deployed (Figure 3(a)). Parasitism was observed one week later at the 3 and $15 \mathrm{~km}$ control sites but with a significantly lower level of parasitism $\left(F_{2,592}=64.69, P<.0001\right.$; Figure $\left.3(\mathrm{a})\right)$. Similarly, four weeks after parasitoid bags were deployed, the level of parasitism of the MHM by H. hebetor remained significantly higher $\left(F_{2,554}=61.02, P<.0001\right)$ at the release site, and the $3 \mathrm{~km}$ locations had significantly higher parasitism than the control located $15 \mathrm{~km}$ away from the release sites (Figure 3(a)). The same trend was observed 35 days post releases $\left(F_{2,607}=77.56\right.$, $P<.0001$; Figure 3(a)).

In Niger in 2010, parasitism was observed at all distances 14 days after parasitoid bags were deployed, but with a significantly higher level of parasitism at the release sites $\left(F_{3,596}=14.81 ; P<.00001\right.$; Figure 3(b)). The same trend was observed 21 days post-parasitoid deployment $\left(F_{3,596}=28.40 ; P<.00001\right.$; Figure 3(b)). However, after 28 days, sites at both 0 and $3 \mathrm{~km}$ distance from the release sites had a similar level of MHM parasitism with $H$. hebetor, and these sites had higher parasitism rates than the control $\left(F_{3,796}=312.93\right.$; $P<.00001$; Figure 3(b)).

In Burkina Faso in 2011, parasitism of the MHM by H. hebetor was observed 14 days after parasitoid bags were deployed in millet farms, with significantly higher parasitism in villages located at the release sites $\left(F_{3,490}=17.22, P<.0001\right.$; Figure $\left.4(\mathrm{a})\right)$. Parasitism increased over the weeks at all locations and remained significantly higher at release sites after 21 days $\left(F_{3,1077}=44.52, P<.0001\right.$; Figure $\left.4(\mathrm{a})\right)$ and 28 days $\left(F_{3,1111}=39.00\right.$, $P<.0001$; Figure $4(\mathrm{a}))$. At the end of the season, all villages located up to $3 \mathrm{~km}$ from

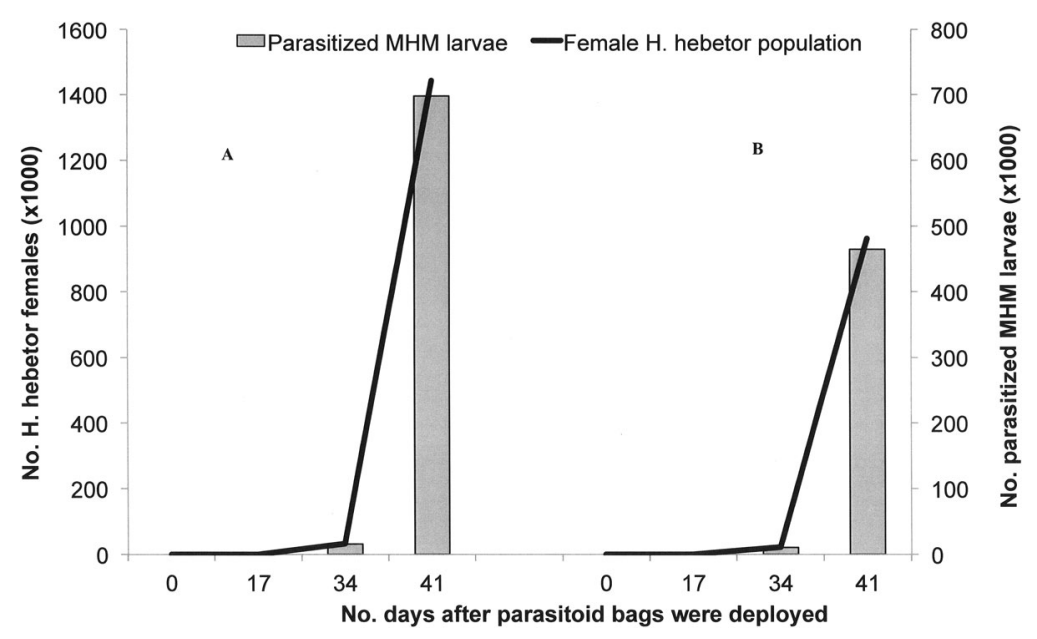

Figure 2. Projected numbers of Habrobracon hebetor (Say) population after augmentative releases and corresponding number of parasitised millet head miner (MHM) larvae in pearl millet fields in Burkina Faso (A) and Niger (B). 

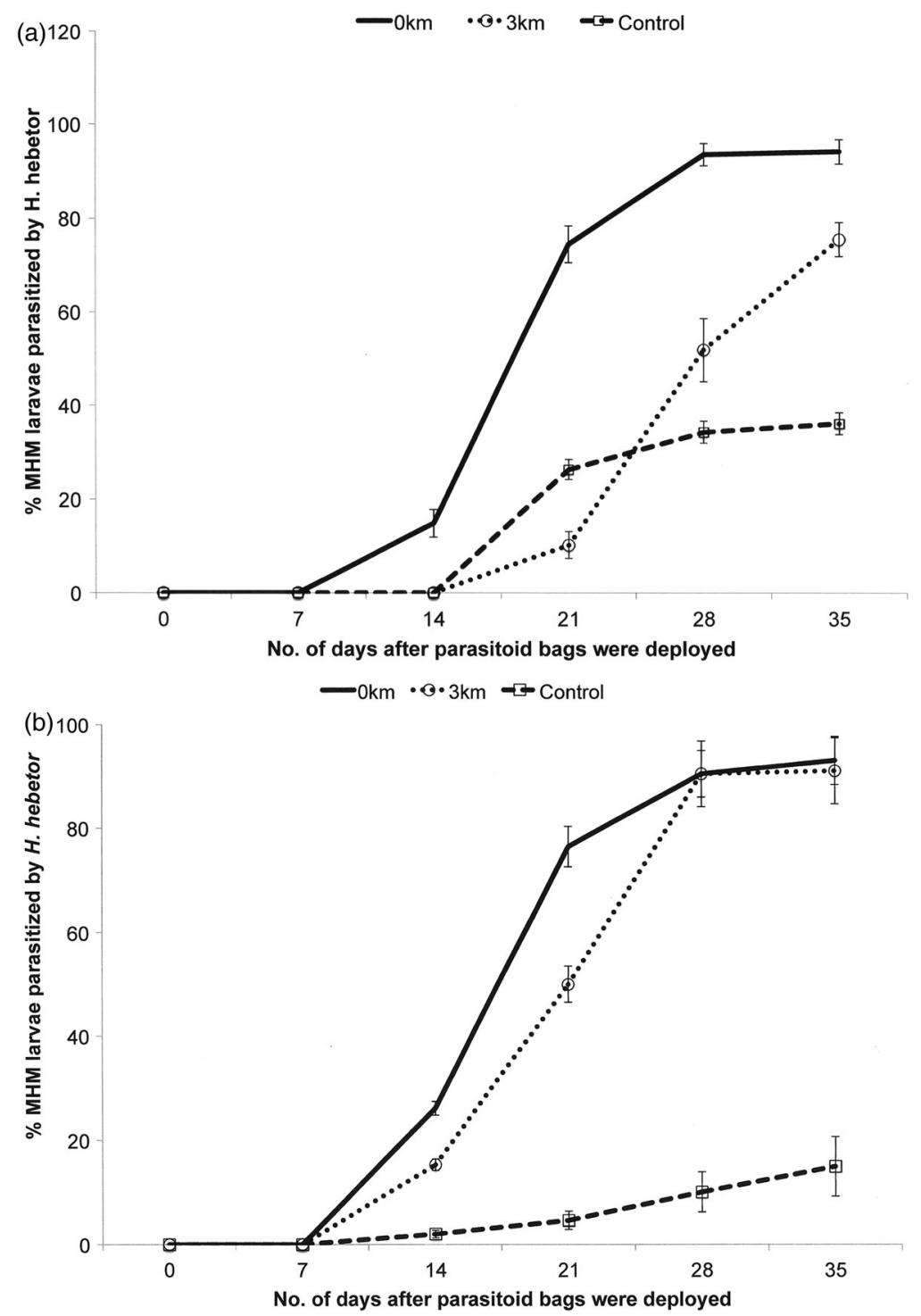

Figure 3. Parasitised larvae of millet head miner (MHM), $H$. albipunctella (\% mean $\pm \mathrm{SE}$ ) due to $H$. hebetor at a different distance from release sites and control villages after augmentative releases of $H$. hebetor in 2010 in (a) Burkina Faso and (b) Niger. (For each date, means were compared by a Student-Newman-Keuls test at the $5 \%$ level.)

parasitoid release sites had over $50 \%$ levels of parasitism and remained significantly higher than the 5- and 15-km control $\left(F_{3,815}=56.21, P<.0001\right.$; Figure $\left.4(\mathrm{a})\right)$.

In Niger in 2011, parasitism was also first recorded two weeks after parasitoid bags were deployed in millet farms, with significantly higher parasitism at release villages $\left(F_{3,596}=\right.$ 30.08, $P<.00001$; Figure 4(b)). From three weeks after release onward, similar levels of parasitism were recorded at the 0 and $3 \mathrm{~km}$ distances, but levels were significantly higher at these sites than at the $5 \mathrm{~km}$ and control sites (three weeks: $F_{3,596}=4.65$, $P=.003$; four weeks: $F_{3,1196}=7.23 ; P<.0001$; Figure $\left.4(\mathrm{~b})\right)$. 

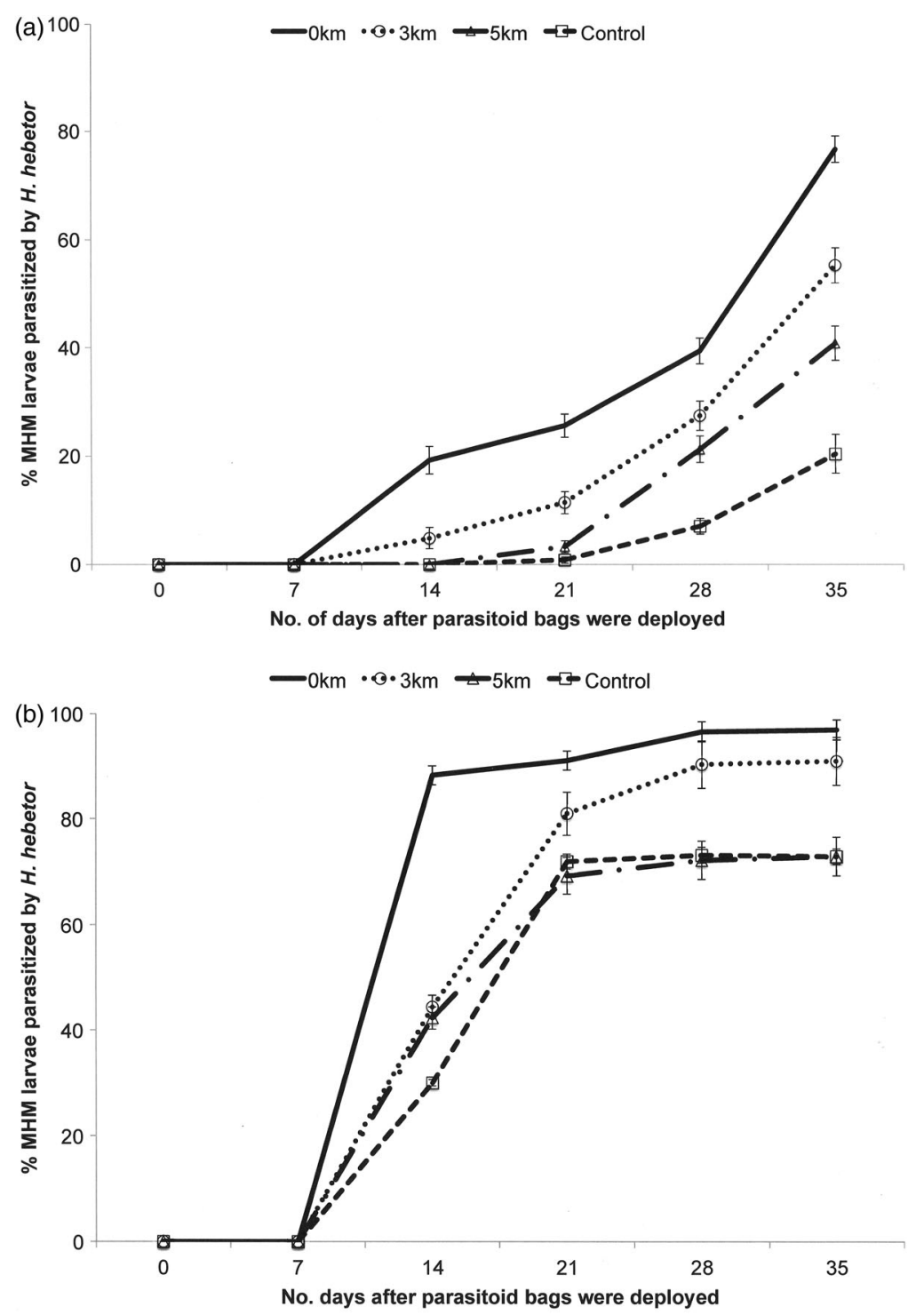

Figure 4. Parasitised larvae of millet head miner (MHM), H. albipunctella (\% mean $\pm \mathrm{SE}$ ) due to $H$. hebetor at a different distance from release sites and control villages after augmentative releases of $H$. hebetor in 2011 in (a) Burkina Faso and (b) Niger. (For each date, means were compared by a Student-Newman-Keuls test at the $5 \%$ level.)

\section{Discussion}

In this study, dispersal of the MHM parasitoid H. hebetor was evaluated in the field after augmentative releases. The parasitoid started emerging from the jute bags one week after being deployed in farmer fields, and each bag delivered 60-70 offspring. This is consistent with previous lab data on parasitoid emergence from jute bags (Ba et al., 2014). The initial population of 60-70 parasitoids per bag corresponds to approximately 900-1000 per release. Based on published life table data of $H$. hebetor (Youm \& Gilstrap, 1993a), this 
initial inoculum could lead to an estimated 1.4 million progenies after one and a half months. This numbers should, however, be lowered because as reported in other settings parasitoid wasps may face predation of generalist predators (Heimpel, Rosenheim, \& Mangel, 1997; Snyder \& Ives, 2001; Traugott, Bell, Raso, Sint, \& Symondson, 2012). Moreover, the few insecticides sprayed on cowpea (Murdock et al., 2008), which is usually intercropped with pearl millet, can also reduce parasitoids population. Such mortality can hamper the parasitism achieved by released $H$. hebetor, hence reducing the efficacy of augmentative biological control. However, our field data indicate high mortality of the MHM due to $H$. hebetor suggesting limited mortality of $H$. hebetor over the cropping season.

Indeed in both countries, and for both 2010 and 2011 years, on-farm parasitism of the MHM by $H$. hebetor was recorded one week after parasitoids began emerging from jute bags (i.e. 14 days after bags were placed). Parasitism increased steadily over the five weeks, with significantly higher parasitism rates in farms located at release sites. However, after 3-4 weeks, very high levels of parasitism were found at all locations up to $3 \mathrm{~km}$ from release points. Usually after 4-5 weeks, parasitism at villages $5 \mathrm{~km}$ from the release sites also reached high levels, but these levels were still lower than levels at the points of release. Parasitoid flight distance varies among species and ecological habits. Trichogrammatoidae, for example, usually disperse only a few metres from release points (Bueno, Parra, \& Bueno, 2012; Gardner, Wright, Kuhar, Pitcher, \& Hoffmann, 2012). Other parasitoid species may disperse only a few metres from release points (Evans, Bolshakova, \& Carlile, 2015; Zappala et al., 2012 ) to a few kilometres (Grillenberger, Gadau, Bijlsma, Van De Zande, \& Beukeboom, 2009). In Brazil, the related Braconidae species, Diachasmimorpha longicaudata, has also been found dispersing lesser distances in coffee plantations (Camargos et al., 2016), while another braconidae species, Microplitis mediator (Haliday), was found to fly a distance of 6-18 km in just a few hours (Yu, Zhang, Wu, Wyckhuys, \& Guo, 2009). In the USA, the related Peristenus braconid parasitoid was found to travel 16-18 km per year after introduced releases (Day, Tilmon, Romig, Eaton, \& Murray, 2000; Pickett et al., 2013).

Most studies of $H$. hebetor refer to insect pests of stored commodities (Ghimire \& Phillips, 2010). Apart from the ongoing work on the use of $H$. hebetor against the MHM in the Sahel (Ba et al., 2014, 2013; Baoua et al., 2014; Kabore et al., 2017), outdoor releases of $H$. hebetor are quite rare, with only a few cases reported in Iran against Helicoverpa armigera larvae in cotton fields (Navaei, Taghizadeh, Javanmoghaddam, Oskoo, \& Attaran, 2002) and in India for the suppression of coconut head caterpillar Opisina arenosella (Desai, Nagwekar, Patil, \& Narangalkar, 2007; Mohanty et al., 2000). Unfortunately, none of those reports investigated field dispersal of $H$. hebetor after release. To our knowledge, the only available study on field dispersal of $H$. hebetor reported a $1 \mathrm{~km}$ dispersal from the release point after 6 days in a tomato field in Russia (Adashkevich, Saidova, \& Takanaev, 1986). This $1 \mathrm{~km}$ dispersal distance is, however, shorter than the $3-5 \mathrm{~km}$ distance found in the current study. This could be due to differences in methodology as Adashkevich et al. (1986) investigated $H$. hebetor dispersal after only 6 days of observations while in the present study $H$. hebetor dispersal was monitored for 5 weeks. Moreover, as reported in other settings, parasitoid species dispersal distance could be influenced by weather, especially temperature (Fahrner, Lelito, \& Aukema, 2015; Yu et al., 2009), wind (Desouhant, Driessen, Lapchin, Wielaard, \& Bernstein, 2003; Kristensen, Schellhorn, Hulthen, Howie, \& Barro, 2013; Messing, Klungness, \& Purcell, 1994) and landscape (Josso et al., 2013). 
As $H$. hebetor is endemic to the Sahel region (Bhatnagar, 1987; Gahukar et al., 1986; Nwanze \& Harris, 1992), the effects of our releases could only be compared to control sites (i.e. $15 \mathrm{~km}$ distance), which had similar levels of MHM infestation, and are not subjected to augmentative releases. Differences in levels of parasitism, especially recorded early in the season, could then be attributed to our augmentative releases. H. hebetor naturally parasitises the MHM at rates ranging from $2 \%$ to $95 \%$, but often towards the end of the season after the crop has been damaged (Bhatnagar, 1989; Gahukar et al., 1986; Youm \& Gilstrap, 1993b). In our case, there is strong evidence of the rapid spread of H. hebetor in pearl millet field, covering a radius of $3 \mathrm{~km}$. However, comparable levels of MHM parasitism with $H$. hebetor were also observed at farther distances $(3-5 \mathrm{~km})$ from release points 3-5 weeks after releases were made. Since $H$. hebetor has a rapid developmental time and a high rate of population growth (Magro \& Parra, 2001; Nikam \& Pawar, 1993), about three generations could develop on larvae of MHM within 7 weeks. This suggests that dispersal of the parasitoid up to $5 \mathrm{~km}$ occurred over several generations, with successive $H$. hebetor generations colonising additional areas. A similar observation was made in Ireland with the Encyrtidae parasitoid wasp, Psyllaephagus pilosus (Noyes), on the psyllid host, Ctenarytaina eucalypti (Maskell), in eucalyptus plantations, in which successive generations of the parasitoid spread to up $70 \mathrm{~km}$ from the release point after only 5 months (Chauzat, Purvis, \& Dunne, 2002). The context is different in the Sahel as the host species, $H$. albipunctella, develops only during the rainy season and remains in diapause for more than 10 months (Gahukar et al., 1986). H. hebetor thus has limited options for survival during the off-season (Kabore et al., 2017).

Parasitoid dispersal is typically density dependent (Antolin \& Strong, 1987). In our case, however, MHM host density was uniform over the area with an estimate of at least $60 \%$ infestation of pearl millet panicles. The village $5 \mathrm{~km}$ away from the release site was reached by the parasitoid almost toward the end of the season. Our findings confirm the effectiveness of augmentative releases of $H$. hebetor for controlling the MHM in the Sahel (Ba et al., 2014, 2013; Baoua et al., 2014; Kabore et al., 2017). As shown in our study, parasitism of MHM larvae by $H$. hebetor increases over several weeks, and the parasitism rates at points of release were much higher than all other distances, with a 1.27-6.20-fold increase in control as observed previously (Ba et al., 2014, 2013).

We were also able to document the seasonal variability of the parasitism at different distances from release points, which was missing in the Garba and Gaoh (2008) study. Our findings clearly demonstrate that the end of seasonal parasitism at $5 \mathrm{~km}$ never reached the level observed at the point of parasitoid releases. The Garba and Gaoh (2008) $5 \mathrm{~km}$ dispersal distance was based on observations made 30 days after parasitoid releases and lack control villages. As indicated earlier, $H$. hebetor had endemic presence in the region and, as reported by Gahukar et al. (1986), H. hebetor is active mainly towards the end of the crop season. For this reason, unless compared with a control, it is not accurate to only attribute the parasitism at $5 \mathrm{~km}$ distance to the augmentative releases but rather to a cumulative effect of natural and augmentation. The formulation of 15 bags per village seems to be appropriate for reducing the pest population at the distance of $3 \mathrm{~km}$. At $5 \mathrm{~km}$ distance, the high level of mortality was found only at the end of the season, which will not provide acceptable control of MHM. For the highest and timely control of the MHM, we suggest the release of parasitoids every $3 \mathrm{~km}$. Since production of parasitoids and release efforts may be economically challenging, releases every $5 \mathrm{~km}$ could 
be used especially for resource-poor communities. Direct release of adult wasps could be investigated to potentially avoid the two-week delay in parasitoid dispersal that results from rearing them in bags; or bags could be deployed two weeks earlier than the required release. Similarly, the number of parasitoids to be released per area needs further investigation.

\section{Acknowledgements}

The opinions expressed herein are those of the authors and do not necessarily reflect the views of the McKnight Foundation. The authors are also grateful to field technicians, Mayaki Gaya, Ousmane Bakoye, Laouali Karimoune, Sabiou Ibrahim, Ouedraogo Theodore and Tarpidiga Simon for their assistance in data collection and to farmers who graciously allow collecting data in their millet fields.

\section{Disclosure statement}

No potential conflict of interest was reported by the authors.

\section{Funding}

This study was made possible through the support provided to INRAN and INERA by the Collaborative Crop Research Program (CCRP) of the McKnight Foundation, Minneapolis, MN [grant numbers 09-036-039].

\section{References}

Adashkevich, B. P., Saidova, Ė, \& Takanaev, A. A. (1986). Migration of Habrobracon. Zashchita Rasteniǔ, 7, 35-36.

Antolin, M., \& Strong, D. (1987). Long-distance dispersal by a parasitoid (Anagrus delicatus, Mymaridae) and its host. Oecologia, 73, 288-292.

Ba, N. M., Baoua, I. B., Kabore, A., Amadou, L., Oumarou, N., Dabire-Binso, C., \& Sanon, A. (2014). Augmentative on-farm delivery methods for the parasitoid Habrobracon hebetor Say (Hymenoptera: Braconidae) to control the millet head miner Heliocheilus albipunctella (de Joannis) (Lepidoptera: Noctuidae) in Burkina Faso and Niger. BioControl, 59(6), 689-696.

Ba, N. M., Baoua, I. B., N’Diaye, M., Dabire-Binso, C., Sanon, A., \& Tamo, M. (2013). Biological control of the millet head miner Heliocheilus albipunctella in the Sahelian region by augmentative releases of the parasitoid wasp Habrobracon hebetor: effectiveness and farmers' perceptions. Phytoparasitica, 41(5), 569-576.

Baoua, I. B., Amadou, L., Oumarou, N., Payne, W., Roberts, J. D., Stefanova, K., \& Nansen, C. (2014). Estimating effect of augmentative biological control on grain yields from individual pearl millet heads. Journal of Applied Entomology, 138(4), 281-288.

Bhatnagar, V. S. (1987). Conservation and encouragement of natural enemies of insect pest in dryland subsistence farming: Problems, progress and prospects in the Sahelian zone. International Journal of Tropical Insect Science, 8(4-6), 791-795.

Bhatnagar, V. S. (1989). Lutte biologique contre la chenille mineuse de l'épi. Sahel PV INFO, 12, 5-9.

Bueno, R. C. O. F., Parra, J. R. P., \& Bueno, A. F. (2012). Trichogramma pretiosum parasitism and dispersal capacity: A basis for developing biological control programs for soybean caterpillars. Bulletin of Entomological Research, 102(1), 1-8.

Camargos, M. G., Alvarenga, C. D., Giustolin, T. A., Paranhos, B. A. J., Oliveira, P. C. C., \& Rabelo, M. M. (2016). Dispersal capacity of fruit fly parasitoid Diachasmimorpha longicaudata (Hymenoptera: Braconidae) in irrigated coffee plantations. Scientia Agricola, 73(3), 227-233. 
Chauzat, M. P., Purvis, G., \& Dunne, R. (2002). Release and establishment of a biological control agent, Psyllaephagus pilosus for eucalyptus psyllid (Ctenarytaina eucalypti) in Ireland. Annals of Applied Biology, 141(3), 293-304.

Day, W. H., Tilmon, K. J., Romig, R. F., Eaton, A. T., \& Murray, K. D. (2000). Recent range expansions of Peristenus digoneutis (Hymenoptera: Braconidae), a parasite of the tarnished plant bug (Hemiptera: Miridae), and high temperatures limiting its geographic distribution in North America. Journal of the New York Entomological Society, 108(3-4), 326-331.

Desai, V. S., Nagwekar, D. D., Patil, P. D., \& Narangalkar, A. L. (2007). Field evaluation of a larval parasite Bracon hebetor Say against coconut black headed caterpillar. Journal of Plantation Crops, 35(3), 188-189.

Desouhant, E., Driessen, G., Lapchin, L., Wielaard, S., \& Bernstein, C. (2003). Dispersal between host populations in field conditions: navigation rules in the parasitoid Venturia canescens. Ecological Entomology, 28(3), 257-267.

Evans, E. W., Bolshakova, V. L. J., \& Carlile, N. R. (2015). Parasitoid dispersal and colonization lag in disturbed habitats: Biological control of cereal leaf beetle metapopulations. Journal of Applied Entomology, 139(7), 529-538.

Fahrner, S. J., Lelito, J. P., \& Aukema, B. H. (2015). The influence of temperature on the flight capacity of emerald ash borer Agrilus planipennis and its parasitoid, Tetrastichus planipennisi: Implications to biological control. BioControl, 60(4), 437-449.

Gahukar, R. T. (1984). Insect pests of pearl millet in West Africa. Tropical Pest Management, 30(2), 142-147.

Gahukar, R. T. (1989). Pest and disease incidence in pearl millet under different plant density and intercropping patterns. Agriculture Ecosystems and Environment, 26(1), 69-74.

Gahukar, R. T. (1990). Reaction of locally improved pearl millets to three insect pests and two diseases in Senegal. Journal of Economic Entomology, 83(5), 2102-2106.

Gahukar, R. T. (1992). Effect of various fertilizers and rates on insect pest/pearl millet relationship in Senegal. Tropical Agriculture, 69(2), 149-152.

Gahukar, R. T., Guèvremont, T. H., Bhatnagar, V. S., Doumbia, Y. O., Ndoye, M., \& Pierrard, G. (1986). A review of the pest status of the millet spike worm, Rhaguva albipunctella de Joannis (Noctuidae: Lepidoptera) and its management in the Sahel. International Journal of Tropical Insect Science, 7(4), 457-463.

Garba, M., \& Gaoh, N. B. (2008, October). Use of Habrobracon hebetor in biological control of Heliocheilus albipunctella, the pearl millet head miner. Paper presented at the 8th international conference on pests in agriculture organized by the French Association of plant protection, Montpellier.

Gardner, J., Wright, M. G., Kuhar, T. P., Pitcher, S. A., \& Hoffmann, M. P. (2012). Dispersal of Trichogramma ostriniae in field corn. Biocontrol Science and Technology, 22(10), 1221-1233.

Ghimire, M. N., \& Phillips, T. W. (2010). Suitability of different Lepidopteran host species for development of Bracon hebetor (Hymenoptera: Braconidae). Environmental Entomology, 39(2), 449-458.

Grillenberger, B., Gadau, J., Bijlsma, R., Van De Zande, L., \& Beukeboom, L. (2009). Female dispersal and isolation-by-distance of Nasonia vitripennis populations in a local mate competition context. Entomologia Experimentalis et Applicata, 132(2), 147-154.

Heimpel, G. E., Rosenheim, J. A., \& Mangel, M. (1997). Predation on adult Aphytis parasitoids in the field. Oecologia, 110(3), 346-352.

Josso, C., Le Ralec, A., Raymond, L., Saulais, J., Baudry, J., Poinsot, D., \& Cortesero, A. M. (2013). Effects of field and landscape variables on crop colonization and biological control of the cabbage root fly Delia radicum. Landscape Ecology, 28(9), 1697-1715.

Kabore, A., Ba, N. M., Dabire-Binso, C. L., \& Sanon, A. (2017). Field persistence of Habrobracon hebetor (Say) (Hymenoptera: Braconidae) following augmentative releases against the millet head miner, Heliocheilus albipunctella (de Joannis) (Lepidoptera: Noctuidae), in the Sahel. Biological Control, 108, 64-69.

Krall, S., Youm, O., \& Kogo, S. A. (1995). Panicle insect pest damage and yield loss in pearl millet. In K. F. Nwanze \& O. Youm (Eds.), Proceeding of an international consultative workshop on panicle insect pest of sorghum and millet (pp. 135-145). Niamey: ICRISAT Sahelian Centre. 
Kristensen, N. P., Schellhorn, N. A., Hulthen, A. D., Howie, L. J., \& Barro, P. J. (2013). Wind-borne dispersal of a parasitoid: The process, the model, and its validation. Environmental Entomology, 42(6), 1137-1148.

Magro, S. R., \& Parra, J. R. P. (2001). Biology of the ectoparasitoid Bracon hebetor Say, 1857 (Hymenoptera: Braconidae) on seven Lepidopteran species. Scientia Agricola, 58(4), 693-698.

Messing, R. H., Klungness, L. M., \& Purcell, M. F. (1994). Short-range dispersal of mass-reared Diachasmimorpha longicaudata and D. tryoni (Hymenoptera: Braconidae), parasitoids of Tephritid fruit flies. Journal of Economic Entomology, 87(4), 975-985.

Mohanty, J. N., Prakash, A., Rao, J., Pawar, A. D., Patnaik, N. C., \& Gupta, S. P. (2000). Parasitization of the coconut black-headed caterpillar, Opisina arenosella walk. In Puri district of Orissa by field release of hymenopterous parasitoids. Journal of Applied Zoological Researches, 11(1), 17-19.

Murdock, L. L., Coulibaly, O., Higgins, T. J. V., Huesing, J. E., Ishiyaku, M. F., \& Sithole-Niang, I. (2008). Cowpea: Legume grains and forages. In C. Kole \& T. C. Hall (Eds.), A compendium of transgenic crop plants (pp. 23-56). Oxford: Blackwell Publishing.

Navaei, A. N., Taghizadeh, M., Javanmoghaddam, H., Oskoo, T., \& Attaran, M. R. (2002, September). Efficiency of parasitoid wasps, Trichogramma pintoii and Habrobracon hebetor against Ostrinia nubilalis and Helicoverpa sp. on maize in Moghan. Paper presented at the 15th Iranian Plant Protection Congress, Kermanshah.

Nikam, P. K., \& Pawar, C. V. (1993). Life tables and intrinsic rate of natural increase of Bracon hehetor Say (Hym., Braconidae) population on Corcyra cephalonica Staint. (Lep., Pyralidae), a key parasitoid of Helicoverpa armigera Hbn. (Lep., Noctuidae). Journal of Applied Entomology, 115(1-5), 210-221.

Nwanze, K. F., \& Harris, K. M. (1992). Insect pests of pearl millet in West Africa. Review of Agriculture Entomology, 80(12), 1133-1155.

Nwanze, K. F., \& Sivakumar, M. V. K. (1990). Insect pests of pearl millet in Sahelian West Africa-II, Raghuva albipunctella de Joannis (Noctuidae, Lepidoptera): Distribution, population dynamics and assessment of crop damage. International Journal of Pest Management, 36, 59-65.

Payne, W., Tapsoba, H., Baoua, I. B., Ba, N. M., N’Diaye, M., \& Dabire-Binso, C. (2011). On-farm biological control of the pearl millet head miner: Realization of 35 years of unsteady progress in Mali, Burkina Faso and Niger. International Journal of Agricultural Sustainability, 9(1), 186-193.

Pickett, C. H., Nieto, D. J., Bryer, A. J., Swezey, L. S., Stadtherr, M., Wisheropp, D., ... Pitcairn, M. (2013). Post-release dispersal of the introduced lygus bug parasitoid Peristenus relictus in California. Biocontrol Science and Technology, 23(8), 861-871.

SAS. (2003). SAS version 9.1 for Windows. Cary, NC: SAS Institute.

Snyder, W. E., \& Ives, A. R. (2001). Generalist predators disrupt biological control by a specialist parasitoid. Ecology, 82(3), 705-716.

Traugott, M., Bell, J. R., Raso, L., Sint, D., \& Symondson, W. O. (2012). Generalist predators disrupt parasitoid aphid control by direct and coincidental intraguild predation. Bulletin of Entomological Research, 102(2), 239-247.

Youm, O., \& Gilstrap, F. (1993a). Life fertility table of Bracon hebetor Say (Hymenoptera: Braconidae) reared on Heliocheilus albipunctella (Lepidoptera: Noctuidae). International Journal of Tropical Insect Science, 14(4), 455-459.

Youm, O., \& Gilstrap, F. (1993b). Population dynamics and parasitism of Coniesta (Haimbachia) ignefusalis, Sesamia calamistis, and Heliocheilus albipunctella in millet monoculture. International Journal of Tropical Insect Science, 14(4), 419-426.

Youm, O., \& Owusu, E. O. (1998). Assessment of yield loss due to the millet head miner, Heliocheilus albipunctella (Lepidoptera: Noctuidae) using a damage rating scale and regression analysis in Niger. International Journal of Pest Management, 44(2), 119-121.

Yu, H., Zhang, Y., Wu, K., Wyckhuys, K. A. G., \& Guo, Y. (2009). Flight potential of Microplitis mediator, a parasitoid of various lepidopteran pests. BioControl, 54(2), 183-193.

Zappala, L., Campolo, O., Grande, S. B., Saraceno, F., Biondi, A., Siscaro, G., \& Palmeri, V. (2012). Dispersal of Aphytis melinus (Hymenoptera: Aphelinidae) after augmentative releases in citrus orchards. European Journal of Entomology, 109, 561-568. 